\title{
ANÁLISE TEÓRICA SOBRE A INFLUÊNCIA DA ESTRUTURA ELETRÔNICA NAS PROPRIEDADES MAGNÉTICAS DOS ELEMENTOS TERRAS-RARAS
}

\author{
F. H. S. Sales' e F. S. H. T. Pinheiro ${ }^{2}$ \\ 'Laboratório de Inovação em Aplicações Física - Instituto Federal do Maranhão \\ fsales@ifma.edu.br - sheldakell@yahoo.com.br
}

Artigo submetido em julho/2011 e aceito em julho/2013

\section{RESUMO}

Apresentamos um estudo teórico que propõe uma abordagem de fácil entendimento sobre as propriedades magnéticas dos Terras-Raras (TR) disprósio (Dy) e o hólmio (Ho), a partir da estrutura eletrônica destes elementos. Os elétrons desemparelhados nos orbitais $4 \mathrm{f}$ dão origem magnetismo dos TR, fazendo com que estes apresentem altos valores de momento magnéticos comparados a outros elementos químicos. Sistemas nanomagnéticos artificiais envolvendo nanocamadas de Dy e Ho tem sido amplamente estudado nos últimos anos, devido principalmente às fases magnéticas exóticas apresentadas por esses materiais, favorecendo, cada vez mais, o aparecimento de novas propriedades magnéticas nestes sistemas, e o interesse da indústrias da magneto-eletrônica e refrigeração magnética.

PALAVRAS-CHAVE: Terras-Raras, Magnetismo, Estrutura Eletrônica.

\section{THEORETICAL ANALYSIS OF THE INFLUENCE OF ELECTRONIC STRUCTURE ON MAGNETIC PROPERTIES OF RARE-EARTH ELEMENTS.}

\begin{abstract}
We present a theoretical study proposes an approach for easy understanding of the magnetic properties of rare-earth (TR) dysprosium (Dy) and holmium (Ho) from the electronic structure of these elements. The unpaired electrons in the $4 \mathrm{f}$ orbitals give rise magnetism of the $T R$, so that they present high values of magnetic moment compared to other chemicals. Nanomagnetic
\end{abstract}

systems involving artificial nanolayers of Dy and Ho have been studied extensively in recent years, due mainly to the exotic magnetic phases presented by these materials, favoring, increasingly, the emergence of new magnetic properties in these systems, industries and interest of the magneto-electronic and magnetic refrigeration.

KEYWORDS: Rare earths, Magnetism, Electronic Structure. 


\section{ANÁLISE TEÓRICA SOBRE A INFLUÊNCIA DA ESTRUTURA ELETRÔNICA NAS PROPRIEDADES MAGNÉTICAS DOS ELEMENTOS TERRAS-RARAS}

\section{INTRODUÇÃO}

Os elementos TR pertencem à série dos lantanídeos formada por quatorze elementos químicos. Esta série é caracterizada pela presença de elétrons $4 \mathrm{f}$. A configuração eletrônica destes elementos pode ser descrita através da configuração eletrônica do Xenônio (Xe) considerando a presença de $\mathrm{N}$ elétrons $\mathrm{f}$, isto é: $[X e] 4 f^{N} 5 d^{0} 6 s^{2}$ ou $[X e] 4 f^{N-1} 5 d^{1} 6 s^{2}$. As TR possuem geralmente valência +3 . Seus orbitais $5 s$ e $4 d$ blindam os elétrons fazendo com que estes não participem das ligações químicas. Mas, se o íon encontrase em meio sólido, ocorrerão transporte de um dos elétrons $f$ da camada de condução para a camada externa $5 d$ de valência. Quando o estado de oxidação atinge o nível +3 , o elemento químico passa então para a configuração eletrônica $[\mathrm{Xe}] 4 \mathrm{f}^{\mathrm{N}-1} \mathrm{~d}^{0} 6 \mathrm{~s}^{0}$. Mesmo nesta nova configuração, os elétrons $\mathrm{f}$ continuam blindados pelas subcamadas $5 s^{2}$ e $5 p^{6}$, não participando mais uma vez das ligações químicas, mas contribuindo para as propriedades magnéticas e ópticas de seu elemento [1].

Pelo "Princípio da Incerteza"de Heisenberg, não é possível afirmar a existência definitiva de uma órbita para o elétron. Adequadamente considera-se a existência de regiões chamadas orbitais, localizadas ao redor do núcleo atômico e com probabilidade máxima de ser encontrado um elétron [2].

Através das soluções da equação de Schrodinger que descrevem o comportamento do elétron ao redor do núcleo, cada elétron pode ser caracterizado pelo seu nível energético. Este procedimento é realizado através dos números quânticos [2] (Tabela 1) principal (n), secundário ou azimutal (I), magnético $\left(m_{l}\right)$ e spin $\left(m_{s}\right)$, sendo que:

1. $\mathrm{n}$ indica o nível energético do elétron, $\operatorname{com} \mathrm{n}=1,2,3, \ldots 14$.

2. l está associado ao sub-nível energético do elétron.

3. $m_{l}$ está associado ao orbital com máxima probabilidade de encontrar o elétron, sendo que cada orbital só pode ser ocupado por 2 elétrons (Tabela 2) (Princípio de Exclusão de Pauli). Estes orbitais estão relacionados com os subníveis $l$, por isso os valores de $m_{l}$ variam de $+\mathrm{l}$ a -l [3].

4. O spin $\mathrm{s}$ (ou $m_{s}$ ) está relacionado à rotação do elétron e é utilizado na distinção dos elétrons de um mesmo orbital (Figura 1), sendo que em um deles atribui-se arbitrariamente o valor $+1 / 2$ e ao outro $-1 / 2$, representados simbolicamente por $\uparrow$ e $\downarrow$, respectivamente, (Tabela 2.).

Tabela 1 - Subníveis energéticos para valores de I.

\begin{tabular}{c|c|c|c|c|c|c|c|c}
\cline { 2 - 7 }$l$ & 0 & 1 & 2 & 3 & 4 & 5 & 6 & 7 \\
\hline Subnível & S & P & D & F & G & H & I & K \\
\hline
\end{tabular}

Tabela 2 - Valores de $\mathrm{m}_{1}$ para o $\mathrm{Dy}^{+3}$.

\begin{tabular}{c|c|c|c|c|c|c|c}
\cline { 3 - 7 }$m_{l}$ & -3 & -2 & -1 & 0 & +1 & +2 & +3 \\
\hline Representação gráfica dos orbitais & $\uparrow \downarrow$ & $\uparrow \downarrow$ & $\uparrow$ & $\uparrow$ & $\uparrow$ & $\uparrow$ & $\uparrow$ \\
\hline
\end{tabular}



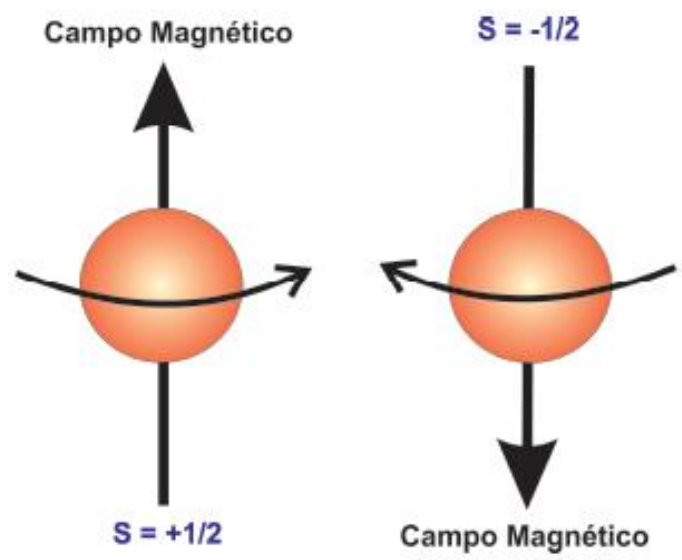

Figura 1 - Representação esquemática do spin s nos sentidos horário e anti-horário, gerando campo magnético nas direções $(s=+1 / 2)$ e $(s=-1 / 2)$, respectivamente.

A Figura 2 apresenta uma representação esquemática visual dos orbitais s, p, d e f.
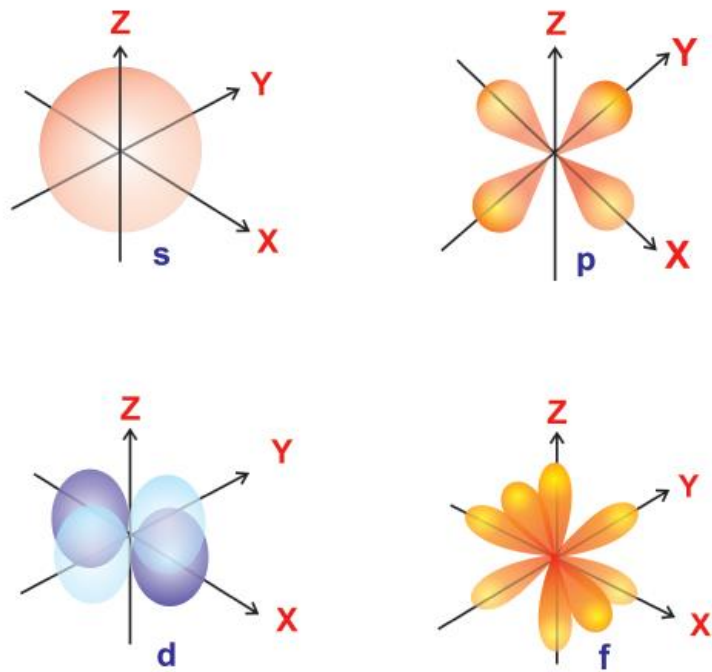

Figura 2 - Representação esquemática dos orbitais s, p, d e f.

\section{MODELAGEM TEÓRICA}

Através da Regra de Hund pode-se determinar o estado fundamental de um íon, cujo número de estados eletrônicos seja permitido $[4,5]$. Esta regra estabelece que:

1. Regra de multiplicidade máxima: "O termo espectroscópico com o maior valor de spin total S, isto é, com mais alto $(2 \mathrm{~S}+1)$ (multiplicidade de spins) corresponderá à mais baixa energia."

2. Se houver mais de um termo com a mesma multiplicidade de spins, o termo com o maior valor de momento angular total $\mathrm{L}$ será o com menor energia.

3. Para uma camada eletrônica com menos da metade do número de elétrons $\mathrm{J}$ será dado por $\mid \mathrm{L}-$ $\mathrm{S} \mid$, e para a camada com mais da metade do número de elétrons, J será $|\mathrm{L}+\mathrm{S}|$. 
De uma forma geral, as propriedades magnéticas dos TR são determinadas quase que exclusivamente pelo estado fundamental de cada elemento. Com base nas regras de Hund e de Pauli, os estados fundamentais de energia para os íons $\mathrm{Dy}^{+3} \mathrm{e} \mathrm{Ho}^{+3}$ podem ser caracterizados.

Na Figura 3 observa-se esquematicamente a distribuição eletrônica do elemento $\mathrm{Dy}^{+3}$, assim como a blindagem dos elétrons da camada 4 f pelos orbitas mais externos 5 s e $5 p$.

$$
\begin{array}{llll}
\mathbf{1} s^{2} & & & \\
\mathbf{2} s^{2} & \mathbf{2} p^{6} & & \\
\mathbf{3} s^{2} & \mathbf{3} p^{6} & \mathbf{3} d^{10} & \\
\mathbf{4} s^{2} & \mathbf{4} p^{6} & \mathbf{4} d^{10} & \mathbf{4} f^{9} \\
\mathbf{5} s^{2} & \mathbf{5} p^{6} & \mathbf{5} d^{0} & \\
\mathbf{6} s^{0} & & &
\end{array}
$$

Figura 3 - Distribuição eletrônica do elemento $\mathrm{Dy}^{+3}$.

Através das regras de Hund, $S, L$ e $J$ dos íons $D^{+3}$ foram determinados analiticamente da seguinte maneira:

$$
\begin{gathered}
S=\sum m_{s}=1 / 2+1 / 2+1 / 2+1 / 2+1 / 2=5 / 2 \text {, entretanto, }(2 \mathrm{~S}+1)=6 . \\
L=\sum m_{l}=+3+2+1+0-1=+5, \text { assim ele está no estado } \mathbf{H} . \\
\mathrm{J}=(2 \mathrm{~S}+\mathrm{L})=15 / 2 .
\end{gathered}
$$

permitindo representar o estado fundamental do $\mathrm{Dy}^{+3}$ na forma ${ }^{2 S+1} \mathrm{X}_{\mathrm{J}}$, onde $\mathrm{X}_{\mathrm{J}}$ corresponde ao momento angular J, na forma ${ }^{6} \mathrm{H}_{15 / 2}$. Com este mesmo raciocínio lógico é fácil chegar ao estado fundamental do íon $\mathrm{Ho}^{+3}$ representado simbolicamente por ${ }^{5} \mathrm{I}_{8}$. Os níveis de energia dos íons TR são constituídos por estados excitados e fundamentais para cada íon [3]. Os estados excitados de quase todos os íons estão separados de seus respectivos estados fundamentais, isso devido ao acoplamento spin-órbita, tornando os estados excitados inacessíveis termicamente.

\section{RESULTADOS E DISCUSSÕES}

A Tabela 3 ilustra algumas propriedades físicas dos lantanídeos tais como o número atômico $Z$ de cada elemento químico, o nível de ocupação dos elétrons nos orbitais $4 \mathrm{f}$, o momento angular orbital total $\mathrm{L}$, o spin total $\mathrm{S}$ e o momento angular total determinado J dos elétrons $4 \mathrm{f}$ e os fatores de Landé g e de Gennes igual a $(g-1) 2 J(J+1)$. 
As TR apresentam propriedades químicas e físicas semelhantes, devido principalmente, às suas configurações eletrônicas. A configuração eletrônica $6 \mathrm{~s}^{2}$, por exemplo, é comum em átomos neutros, assim como a ocupação do orbital $4 f$ (exceto o lantânio, que não possui ocupação neste estado fundamental). 0 estado de oxidação trivalente +3 é o mais presente na maioria dos TR.

Tabela 3 - Propriedades eletrônicas para íons trivalentes de terras-raras.

\begin{tabular}{cccccccc} 
Ion $^{3+}$ & $\mathrm{Z}$ & $4 f^{n}$ & $\mathrm{~L}$ & $\mathrm{~S}$ & $\mathrm{~J}=\mathrm{S}+\mathrm{L}$ & $g$ & $(g-1)^{2} J(J+1)$ \\
\hline $\mathrm{La}$ & 57 & 0 & 0 & 0 & 0 & - & 0 \\
$\mathrm{Ce}$ & 58 & 1 & $1 / 2$ & $5 / 2$ & $5 / 2$ & $6 / 7$ & 0.18 \\
$\mathrm{Pr}$ & 59 & 2 & 5 & 1 & 4 & $4 / 5$ & 0.80 \\
$\mathrm{Nd}$ & 60 & 3 & 6 & $3 / 2$ & $9 / 2$ & $8 / 11$ & 1.84 \\
$\mathrm{Pm}$ & 61 & 4 & 6 & 2 & 4 & $3 / 5$ & 3.20 \\
$\mathrm{Sm}$ & 62 & 5 & 5 & $5 / 2$ & $5 / 2$ & $2 / 7$ & 4.46 \\
$\mathrm{Eu}$ & 63 & 6 & 3 & 3 & 0 & - & 0 \\
$\mathrm{Gd}$ & 64 & 7 & 0 & $7 / 2$ & $7 / 2$ & 2 & 15.75 \\
$\mathrm{~Tb}$ & 65 & 8 & 3 & 3 & 6 & $3 / 2$ & 10.50 \\
$\mathrm{Dy}$ & 66 & 9 & 5 & $5 / 2$ & $15 / 2$ & $4 / 3$ & 7.08 \\
$\mathrm{Ho}$ & 67 & 10 & 6 & 2 & 8 & $5 / 4$ & 4.50 \\
$\mathrm{Er}$ & 68 & 11 & 6 & $3 / 2$ & $15 / 2$ & $6 / 5$ & 2.55 \\
$\mathrm{Tm}$ & 69 & 12 & 5 & 1 & 6 & $7 / 6$ & 1.17 \\
$\mathrm{Yb}$ & 70 & 13 & 3 & $1 / 2$ & $7 / 2$ & $8 / 7$ & 0.32 \\
$\mathrm{Lu}$ & 71 & 14 & 0 & 0 & 0 & - & - \\
\hline
\end{tabular}

Em 1966 foi proposto que a projeção do spin em J descrevesse a interação magnética, tendo em vista que nesta época o número quântico J se consolidava como um bom localizador de momentos magnéticos, levando assim a substituição do momento $S$ por (g-1) J no estudo teórico dos TR.

Os momentos de dipolos magnéticos $(\mu)$ efetivamente localizados são originados dos elétrons desemparelhados localizados no orbital $4 \mathrm{f}$. Esses momentos foram determinados, isto é $[6,7]$ :

$$
\vec{\mu}=g \mu_{B} \vec{J}
$$

(Equação 1)

onde $\mathrm{J}$ é o operador momento angular total com autovalor $[\mathrm{J}(\mathrm{J}+1)]^{1 / 2}, \mu_{\mathrm{B}}$ é o magnéton de Bohr $(1 \mu \mathrm{B}=$ $5,79 \times 10^{-2} \mathrm{meV} . \mathrm{T}^{-1}$ ) e g é o fator de Landé, definido como:

$$
g=[S(S+1)-L(L+1)+3 J(J+1)] 2 J(J+1)
$$

que pode ser escrito na forma: 


$$
g=3 / 2+[S(S+1)-L(L+1)] / 2 J(J+1)
$$

Assim, um elétron com momento angular orbital $\mathrm{L}$ e momento angular de spin $\mathrm{S}$ terá um momento magnético de intensidade $\mu$. Analiticamente, o momento magnético para $\circ \mathrm{Dy}^{+3}$ pode ser determinado:

$$
\begin{gathered}
g=3 / 2+[S(S+1)-L(L+1)] / 2 J(J+1) \\
g=3 / 2+[(35 / 4)-30] /(255 / 2)=4 / 3
\end{gathered}
$$

Substituindo este valor na Equação 1 tem-se que:

$$
\mu_{D y}=(4 / 3)[(15 / 2)(17 / 2)]^{1 / 2} \mu_{B} \cong 10.63 \mu_{B}
$$

Da mesma forma, encontra-se o momento magnético do $\mathrm{Ho}^{+3}$, isto é,

$$
\mu_{H o} \cong 10.60 \mu_{B} \text {. }
$$

\section{CONCLUSÕES}

O desemparelhamento de elétrons no orbital $4 \mathrm{f}$ origina o magnetismo nos lantanídeos. Estes elétrons ficam aprisionados nos orbitais mais internos e fechados dos átomos, apresentando fraca contribuição para as ligações químicas. Tal configuração torna os elétrons $4 \mathrm{f}$ íons livres portadores de momento angular total $J$ devido à interação entre os momentos orbital $L$ e de spin $S$, resultando no momento magnético que produz o magnetismo nas TR.

\section{REFERÊNCIAS BIBLIOGRÁFICAS}

[1] LAPOLLI, A. L., Handbook on the physics and chemistry of rare earths: Metals. North-Holland, vol. 1, 1978.

[2] USBERCO, J. and salvador, E., Química volume único, Saraiva, São Paulo, 2002.

[3] COTTON, S., Lanthanide and actinide chemistry, John Wiley, England, 2006.

[4] N. W. Mermin and N. D. Mermin, Solid state physics, College edition, USA, 1976.

[5] SUBRAMANIAN, N. and OLIVEIRA, S. F., Algumas considerações sobre a energia de Hund e a estrutura eletrônica de átomos no ensino de química. Química Nova, vol. 20, pp. 313\{318\}, 1997.

[6] MELLO, V. D., Propriedades magnéticas de filmes finos de Disprósio. Tese de Doutorado, UFRN, 2005.

[7] SALES, F. H. S. Sales, Fases Magnéticas de Sistemas Nanoestruturado de Terras-Raras. Tese de Doutorado, UFRN, 2011. 